\title{
Prototype implementation of an islanding detection relay based on pattern classification of current and voltage transients
}

\author{
N.W.A. Lidula ${ }^{*}$, A.D. Rajapakse ${ }^{2}$, Jean-Paul Pham ${ }^{2}$ and N. Denboer ${ }^{3}$ \\ ${ }^{I}$ Department of Electrical Engineering, Faculty of Engineering, University of Moratuwa, Katubedda, Moratuwa. \\ ${ }^{2}$ Department of Electrical and Computer Engineering, Engineering and Information Technology Complex, University of Manitoba, Winnipeg, Canada. \\ ${ }^{3}$ Trans Grid Solutions Incorporation, Manitoba, Canada.
}

Revised: 25 March 2013; Accepted: 9 May 2013

\begin{abstract}
A prototype islanding detection relay, which uses decision tree classifier to categorize the transient generating events as 'islanding' or 'non-islanding' was implemented and tested. It consisted of two basic stages of signal processing to extract the required feature vectors for the classification. The first stage involved signal filtering and in the second stage signals were processed by rectifying, summing, and lowpass filtering to get the energy content in the three phases during a selected time-frame. A simple radial medium voltage distribution system having a single distributed generator was simulated in PSCAD/EMTDC to obtain the transient waveforms, and the performance of the relay was tested with signals generated using an RTP real-time waveform playback instrument. The experimental results showed high accuracy in detecting islanding events within a response time of less than two cycles. The proposed relay can be implemented with a very low cost and is simple in construction. Therefore, this could be an acceptable low cost substitute for the expensive and complex transfer trip schemes, which are commonly in use with the distributed generation.
\end{abstract}

Keywords: Active filters, decision tree classification, distributed generation, islanding, transients.

\section{INTRODUCTION}

The term distributed generation (DG) is referred to the small-scale generators connected to medium or low voltage power systems. They are not usually centrally planned or dispatched. DGs change the nature of the distribution system from passive to active, giving rise to certain technical issues (Jenkins et al., 2000) such as power-islanding, which is defined as 'a condition in which a portion of the utility system having both loads and distributed energy resources (DER) remains energized while isolated from the remainder of the utility system' (IEEE, 2003). Unintentional islanding results in several safety and power quality issues including abnormal variations in frequency and voltage, possibility of creating ungrounded systems, and potential safety hazards for repair crews.

In formulating guidelines and/or rules for interconnecting DGs in the distribution systems, the most common practice is to adopt the IEEE standard 1547 (IEEE, 2003). These operational practice standards have been developed with an underlying assumption that the power system is radial. In the past, the power available from DGs was not essential to support the load on the system. Hence, the standards suggest to disconnect the DGs for any 'significant' fault (a fault that could require the protection to be provoked) on the distribution system and thus, maintaining the radial nature of the system. Consequently, the islanding detection relays are designed to avoid power islanding, and these relays are referred to as anti-islanding relays (Xu et al., 2004; Mahat et al., 2008).

However, there is a change in the approach towards islanding with the initiatives on microgrids (Chowdhury et al., 2009; Lidula \& Rajapakse, 2011). The performance of the power-islanding detection methods in a microgrid setup is more demanding as the microgrid is designed to operate as a stable power island. Many powerislanding detection methods in practice would find it difficult to meet the qualities demanded by micorgrids (Katiraei et al., 2005) without the use of expensive telecommunication facilities. 
Different islanding detection methods proposed in the literature can be mainly categorized into three groups: passive, active and telecommunication based methods (Xu et al., 2004; Mahat et al., 2008). The passive methods make decisions based on the measured electrical quantities such as voltage and frequency (Kim \& Hwang, 2000; Walling \& Miller, 2002; Funabashi et al., 2003; Freitas et al., 2005a,b). In the active methods, disturbances are injected into the network and islands are detected based on the system responses to the injected disturbance (Redfern et al., 1993; O'Kane \& Fox, 1997; Ropp et al., 1999; Hung et al., 2003; Mahat et al., 2009; Etxegarai et al., 2011). The telecommunication-based methods use reliable communication links to transmit the circuit breaker status signals, which alert and trip the DGs when islands are formed (Redfern et al., 1993; Xu et al., 2004; Etxegarai et al., 2011). Passive islanding detection methods are preferred and widely used over the active and telecommunication based methods due to considerations such as the cost, simplicity and power quality.

In a situation where the intentional islanded operation is considered, there is a higher chance for the load and the generation of the island to be approximately balanced. Some passive islanding detection methods may fail to detect, or take longer time to detect the islands when such power balance exists. On the other hand, with low security levels observed in available passive anti-islanding protection, they cannot be made overly sensitive as that could lead to some unexpected control actions in a microgrid type of setup (Katiraei et al., 2005). Consequently, looking for faster and more reliable power-islanding detection methods, transfer trip schemes, which come under telecommunication based methods are emerging (Etxegarai et al., 2011). There are some recent research carried out using novel technologies such as hybrid methods, wavelets and/or classification techniques like neural networks and decision trees (Salman \& King, 2006; El-Arroudi et al., 2007; Mahat et al., 2009; Lidula \& Rajapakse, 2010).

A hybrid method, which uses an average rate of voltage change to initiate a real power shift that changes the real power of DG, is proposed in Mahat et al. (2009). The proposed method has shown higher accuracy, but the islanding detection time varies from 5 to 25 cycles depending on the power imbalance. In El-Arroudi et al. (2007), the use of decision tree (DT) classifier is investigated for islanding detection. However, the proposed methodology involves some complex set of features including total harmonic distortion of current/ voltage, gradient of the product of voltage and power factor, etc. for classification. It has a misclassification rate of $16.67 \%$ in islanding detection, which gives only $83.33 \%$ islanding detection accuracy. The use of artificial neural network (ANN) for islanding detection has been investigated by Salman et al. (2006) looking at two different architectures. It reports the best achieved accuracy from one of the proposed architecture as $84.44 \%$ and $98-99 \%$ with the other architecture. However, it does not mention whether the faults are considered in the classification. The islanding detection method proposed in Lidula et al. (2010) involves extraction of current and voltage signal energies in different frequency bands through discrete wavelet transform and the use of DT classification. The performed verification tests has shown more than $98 \%$ classification accuracy with $95 \%$ confidence and a response time of less than 2 cycles.

This paper presents detailed information on hardware implementation of an islanding detection method that uses current and voltage transient signals as an extension of Pham et al. (2011). The proposed methodology in this paper uses simple analog band pass filters to extract the features from the current and voltage signals for accurate identification of power islands.

\section{TRANSIENT BASED ISLANDING DETECTION}

The transients generated during an islanding event contain unique signatures. The hypothesis that these unique signatures contained in transient signals can be used to distinguish islanding events from the other disturbances with the help of a suitable classifier, was proved in Lidula et al. (2010). It has been shown that more than $98 \%$ overall classification accuracy can be achieved with $95 \%$ confidence. However, hardware implementation of the above islanding detection technique requires sampling of input signal at $10 \mathrm{kHz}$ and processing that data in real time. This needs high-end digital signal processors (DSP). Such expensive hardware may not be feasible for very small-scale distributed generators.

This paper proposes a novel, simple and cost effective approach for classifying islanding events based on the hypothesis proved in Lidula et al. (2010) essentially by designing a novel simple feature extraction approach.

Figure 1 illustrates the block diagram of the proposed islanding detection approach, which is based on the embedded signatures of transient signals. Figure 1 depicts that islanding detection is completed in three major stages: (1) data collection, (2) feature extraction and (3) classification. At data collection, current and voltage signals are measured at the generator terminal with the 


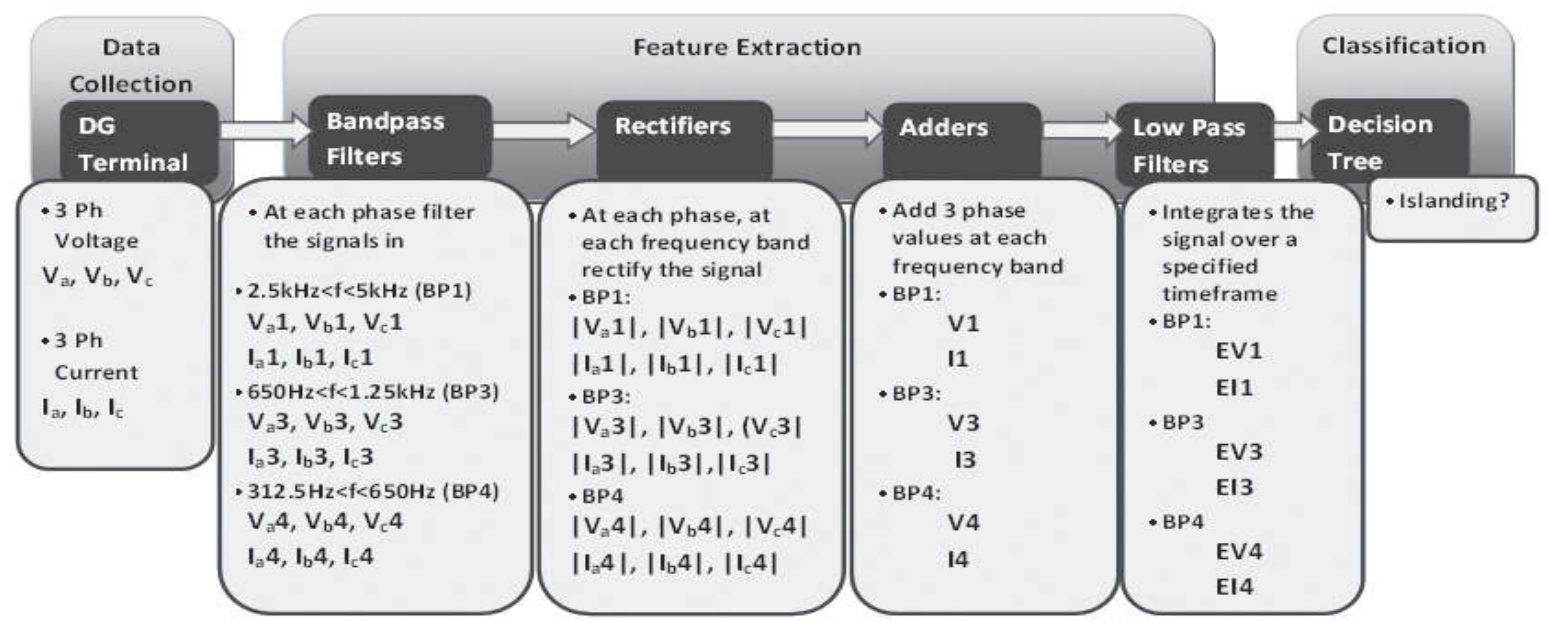

Figure 1: The proposed islanding detection method

use of current and voltage transformers. Although it is proved that transient signals embed unique signatures facilitating the classification of islanding events, they are not directly distinguishable in the measured signals. Therefore, the measured signals need to be pre-processed to extract the required features that assist the process of classification using the decision tree (DT). Instead of using digital technologies, the feature extraction process was designed using simple analog techniques. The use of analog systems not only provides a low cost on implementation, but also gives a low computational cost compared to using feature extraction techniques such as discrete wavelet transform or S-transform in analysing transient signals.

In the proposed feature extraction method, the measured three-phase voltages $\left(\mathrm{V}_{\mathrm{a}}, \mathrm{V}_{\mathrm{b}}, \mathrm{V}_{\mathrm{c}}\right)$ and currents $\left(I_{a}, I_{b}, I_{c}\right)$ at the DG terminal were filtered into three different frequency bands (BP1: $2500-5000 \mathrm{~Hz}, \mathrm{BP} 3$ : 650-1250 Hz and BP4: 312.5-650 Hz) using band-pass filters as shown in Figure 1.The required frequency bands were selected based on Lidula et al. (2010) considering the DWT coefficients used, and further optimized through the process of building the optimal decision tree classifier. The three-phase outputs of current and voltage in each frequency band (i.e. in the frequency band BP1: $I_{a} 1, I_{b} 1, I_{c} 1$ and $V_{a} 1, V_{b} 1, V_{c} 1$ ) were then rectified using full-wave rectifiers (i.e. giving $\left|\mathrm{I}_{\mathrm{a}} 1\right|,\left|\mathrm{I}_{\mathrm{b}} 1\right|,\left|\mathrm{I}_{\mathrm{c}} 1\right|$ and $\left|\mathrm{V}_{\mathrm{a}} 1\right|$, $\left|\mathrm{V}_{\mathrm{b}} 1\right|,\left|\mathrm{V}_{\mathrm{c}} 1\right|$ in BP1) and fed into three input adders, where three phase values were summed together (i.e. in BP1, $\mathrm{V} 1=\left|\mathrm{V}_{\mathrm{a}} 1\right|+\left|\mathrm{V}_{\mathrm{b}} 1\right|+\left|\mathrm{V}_{\mathrm{c}} 1\right|$ and $\left.\mathrm{I} 1=\left|\mathrm{I}_{\mathrm{a}} 1\right|+\left|\mathrm{I}_{\mathrm{b}} 1\right|+\left|\mathrm{I}_{\mathrm{c}} 1\right|\right)$. The lowpass filters were finally used to integrate the adder output in a definite time window creating the required features (i.e. in BP1: EV1 and EI1) for the decision tree classifier. The following subsections briefly discuss the theory relevant to each component used in the implementation.

\section{Band-pass filters}

Fourth-order Butterworth band-pass filters (BPF) were applied in this study. The filters were designed by cascading two second-order BPF that use the multiple feedback (MFB) topology. The MFB topology allows the gain, bandwidth, and mid-frequency to be adjusted independently of one another. The fourth-order MFB band-pass filter topology is shown in Figure 2 (Kugelstadt, 2002). The general equation for the transfer function of a second order Butterworth band-pass filter is given by equation (1).

$H(s)=\frac{\left(A_{m} / Q\right) s}{1+(1 / Q) s+s^{2}}$

With the cascading of two $2^{\text {nd }}$ order BPFs to get a $4^{\text {th }}$ order BPF, the transfer function thus, can be given by equation (2).

$H(s)=\frac{\left(A_{m i} / Q_{i}\right) \alpha s}{\left[1+\left(\alpha s / Q_{i}\right)+(\alpha s)^{2}\right]} \cdot \frac{\left(A_{m i} / Q_{i}\right)(s / \alpha)}{\left[1+\left(s / \alpha Q_{i}\right)+(s / \alpha)^{2}\right]}$

$\alpha^{2}+\left[\frac{\alpha a_{1}}{b_{1} Q\left(1+\alpha^{2}\right)}\right]+\frac{1}{\alpha^{2}}-2-\frac{1}{b_{1} Q^{2}}=0$

where, $\alpha$ is a scaling factor determined through successive approximation of equation (3). $Q_{i}$ and $A_{m i}$ are the quality factor and gain, respectively of both individual $2^{\text {nd }}$ order 
filters. Once $\alpha$ is found, the values of $Q_{i}$ and $A_{m i}$ can be found from equations (4) and (5), respectively. Once $Q_{i}$ and $A_{m i}$ are known, the resistor values are calculated using relationships shown in Table 1, for a pre-defined value of capacitance $(10 \mathrm{nF})$.

$$
\begin{aligned}
& Q_{i}=Q \cdot \frac{\left(1+\alpha^{2}\right) b_{1}}{a_{1}} \\
& A_{m i}=\frac{Q_{i}}{Q} \sqrt{\frac{A_{m}}{b_{1}}}
\end{aligned}
$$

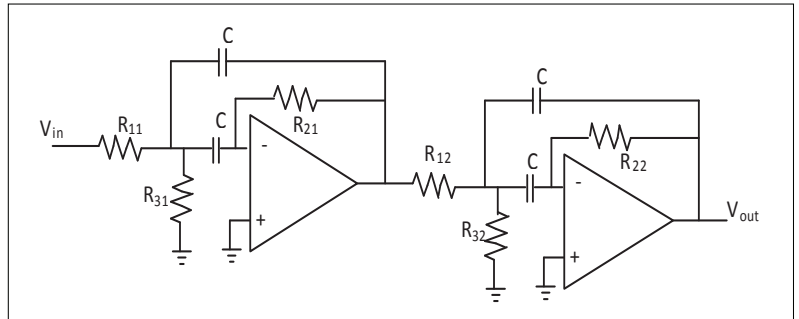

Figure 2: Fourth-order multiple feedback band-pass filter circuit (Kugelstadt, 2002)

Table 1: Relationships: the resistances of the $4^{\text {th }}$ order band-pass filter

\begin{tabular}{cccccc}
\hline $\mathrm{R}_{21}$ & $\mathrm{R}_{11}$ & $\mathrm{R}_{31}$ & $\mathrm{R}_{22}$ & $\mathrm{R}_{12}$ & $\mathrm{R}_{32}$ \\
\hline$\frac{\alpha Q_{i}}{\pi f_{m} C}$ & $\frac{R_{21}}{2 A_{m i}}$ & $\frac{A_{m i} \mathrm{R}_{11}}{2 Q_{i}^{2}+A_{m i}}$ & $\frac{Q_{i}}{\pi \alpha f_{m} C}$ & $\frac{R_{22}}{2 A_{m i}}$ & $\frac{A_{m i} \mathrm{R}_{12}}{2 Q_{i}^{2}+A_{m i}}$ \\
\hline
\end{tabular}

\section{Rectifiers}

After filtering out the waveforms into different frequency bands, the features were formed by calculating the energy content of each filtered signal in a selected time window. The first stage of the energy extraction process is the fullwave rectifier.

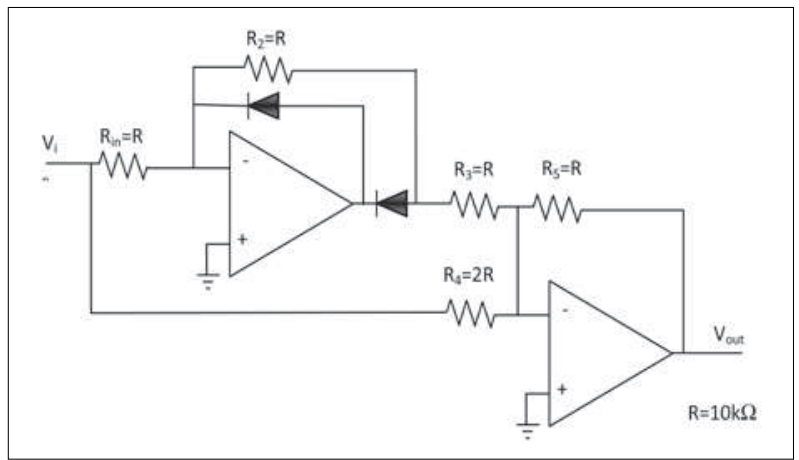

Figure 3: Full-wave rectifier circuit (Gerstenhaber \& Malik, 2010)
The full-wave rectifier shown in Figure 3 (Gerstenhaber $\&$ Malik, 2010) performs the squaring function in the energy extraction process.

\section{Adders}

In order to create a three-phase energy value, the rectified three-phase inputs were summed together using an inverting adder circuit shown in Figure 4. The output of this circuit is given by equation (6). Additionally, the three phase adder circuit allows amplifying the signals by adjusting the ratio of $R_{f} / R$. Thus, the inverting topology effectively supports the cancellation of the negative gain of the next step of the energy extraction process: the low-pass filter.

$V_{\text {out }}=-\frac{R_{f}}{R}\left(V_{A}+V_{B}+V_{C}\right)$

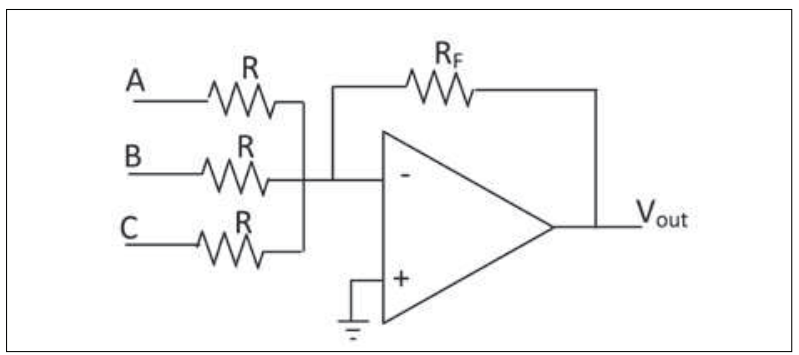

Figure 4: Three-phase adder circuit

\section{Low-pass filters}

The final stage of the energy extraction process is a second-order Butterworth low-pass filter. The low-pass filter performs the integration of the waveform over a definite time window $(0.01 \mathrm{~s})$. The circuit diagram of the second-order low-pass MFB topology is shown in Figure 5. The general transfer function of a low-pass filter is given by equation (7), which gives equation (8) for the MFB low-pass filter shown in Figure 5 (Kugelstadt, 2002).

$H(s)=-\frac{A_{0}}{1+a_{1} s+b_{1} s^{2}}$

$H(s)=-\frac{R_{2} / R_{1}}{1+\omega_{C} C_{1}\left(R_{2}+R_{3}+R_{2} R_{3} / R_{1}\right) s+\omega_{C}{ }^{2} C_{1} C_{2} R_{2} R_{3} s^{2}} \cdots$

For a given cut-off frequency, $\mathrm{f}_{\mathrm{c}}, \mathrm{C}_{1}$ and $\mathrm{C}_{2}$ the resistor values can be calculated using the relationships given 
in Table 2. In selecting $\mathrm{C}_{2}$, it is required to satisfy equation (9) for $\mathrm{R}_{2}$ to be a real value.

$$
\mathrm{a}_{1}^{2} C_{2}^{2}-4 b_{1} C_{1} C_{2}\left(1-A_{0}\right) \geq 0 d
$$

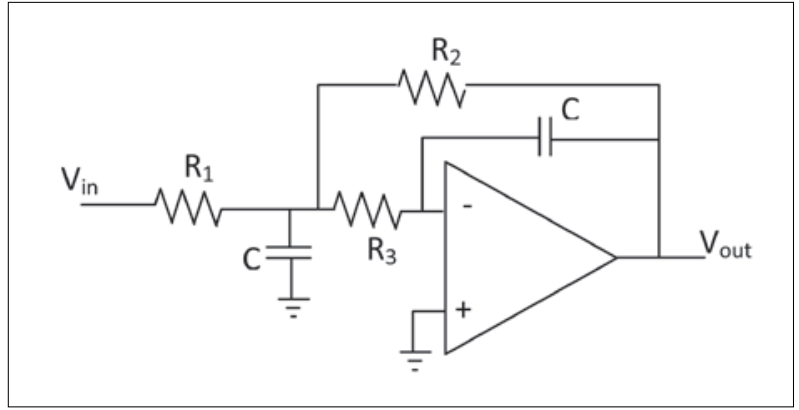

Figure 5. Second-order low-pass filter circuit (Kugelstadt, 2002)

Table 2: Relationships: the resistances of the second order low-pass filter

\begin{tabular}{ccc}
\hline $\mathrm{R}_{1}$ & $\mathrm{R}_{2}$ & $\mathrm{R}_{3}$ \\
$\frac{a_{1} C_{2}-\sqrt{a_{1}^{2} C_{2}^{2}-4 b_{1} C_{1} C_{2}\left(1-A_{0}\right)}}{4 \pi f_{c} C_{1} C_{2}}$ & $\frac{R_{2}}{-A_{0}}$ & $\frac{b_{1}}{4 \pi^{2} f_{c}^{2} C_{1} C_{2} R_{2}}$
\end{tabular}

\section{Decision tree classifier}

A decision tree (DT) is constructed based on the training data, and represented as a binary tree. The DT starts with the 'Root', and it contains the total training dataset. Starting from the 'Root', at each 'Internal Child Node' an attribute is tested and the data is separated to two groups based on the agreement and disagreement with the attribute. The 'Terminal Node' represents the predicted class (Breiman et al., 1984; Steinberg \& Colla, 1995). Figure 6 shows a simple structure of a DT for a two class problem. The CART ® Pro V6 commercial software (Steinberg \& Colla, 1995) was used to develop the decision tree classifier.

CART algorithm generates decision trees based on a splitting rule. The basic idea of the splitting rule is to choose a split (attribute to test) among all the possible splits at each node so that the resulting child nodes are the 'purest'. In the CART algorithm, each split depends on the value of only one predictor variable (input feature). It carries out a brute force search through all possible splits to find the particular split. The 'Gini rule,' which searches for the largest class in the learning dataset to isolate it from the rest of the data was selected as the splitting rule in this study. For a particular split $s$ at node $t$, the Gini impurity measure $i(t)$ is defined as:

$i(t)=1-\sum_{1}^{J} p^{2}\left(Y_{j} / t\right)$

where, $t$ is the node number, $Y_{j}=\operatorname{class}(j=1,2, \ldots . \mathrm{J})$ and $p\left(Y_{j} \mid t\right)$ is the class probability distribution of the dependent variable at node $t$.

Once the impurity measure is calculated, then the goodness of split $s, \Delta i(s, t)$ is calculated using equation (11).

$\Delta i(s, t)=i(t)-p_{L}\left[i\left(t_{L}\right)\right]-p_{R}\left[i\left(t_{R}\right)\right]$

where $p_{L}$ is the proportion of cases at node $t$ that go into the left child node, $t_{L}$ and $p_{R}$ is the proportion of cases at node $t$ that go into the right child node, $t_{R}, i\left(t_{L}\right)$ is the impurity of the left child node and $i\left(t_{R}\right)$ is the impurity of the right child node.

The best split point is the one that maximizes the goodness of split, $\Delta i(s, t)$ when the node is split according to it. The decision tree classification technique is well explained in Breiman et al. (1984), Steinberg \& Colla (1995) and Lidula \& Rajapakse (2010).

CART also provides a ranking of input features based on each variable's contribution to the overall tree. Variable importance is presented by scaling the values relative to the 'best performing variable', which is the variable that minimizes the Gini impurity the most at every node it is considered as a splitter (Breiman et al., 1984; Steinberg \& Colla, 1995; Lidula \& Rajapakse , 2010).

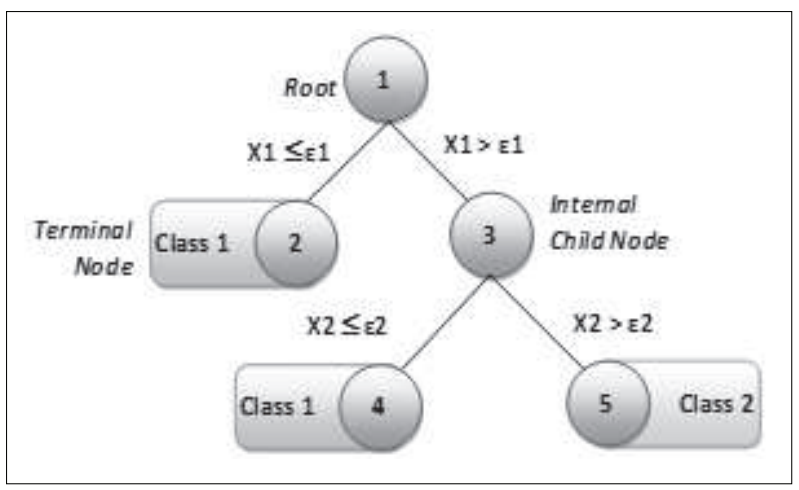

Figure 6: A simple model of a decision tree 


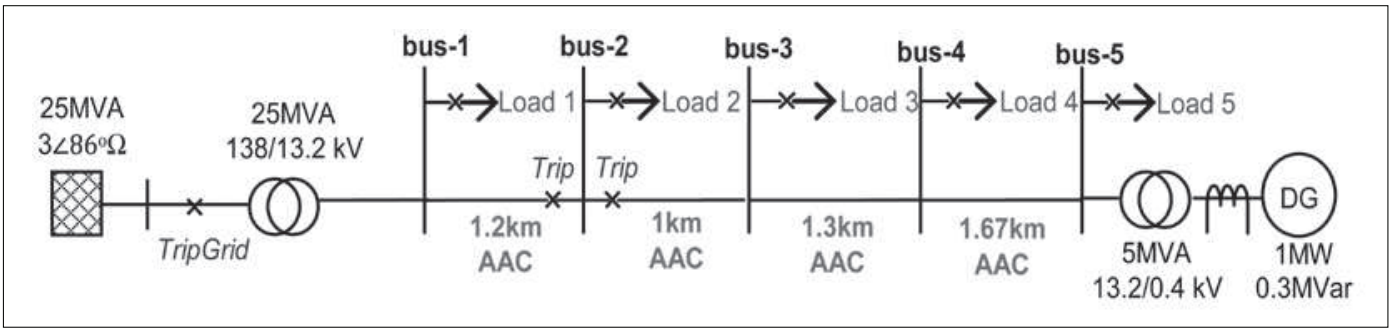

Figure 7: Test system

\section{TEST SYSTEM AND CLASSIFIER MODELLING}

\section{Test system}

The simple radial MV distribution network shown in Figure 7 was simulated using PSCAD/EMTDC power system simulation software.

The distribution network consists of five bus-bars each having lumped loads, and bus-5 has a $1 \mathrm{MW}, 0.3 \mathrm{MVar}$ synchronous generator connected via a $13.2 / 0.4 \mathrm{kV}$ transformer. All loads in the network were simulated as constant RL loads. The nominal system frequency is $60 \mathrm{~Hz}$. It was intended to classify the system disturbances into two classes of events, namely 'non-islanding' and 'islanding'. The non-islanding cases include: (i) normal operation; (ii) switching of loads and (iii) temporary faults on bus-2. The islanding cases simulated include: (i) opening of breaker 'Tripgrid'; (ii) opening of breakers 'Trip' and (iii) opening of breakers 'Trip' following a fault on bus- 2 .

The simulations were carried out under different loading conditions by changing the system total load between $3 \mathrm{MW}$ to $10 \mathrm{MW}$ keeping a power factor close to 0.95. The tripping and fault times were also varied. The cases with faults were simulated for three-phase (ABC), three-phase-to-ground $(\mathrm{ABCG})$, line-to-line $(\mathrm{AB}, \mathrm{AC}$, $\mathrm{BC}$ ), and line-to-ground ( $\mathrm{AG}, \mathrm{BG}, \mathrm{CG}$ ) faults, where the fault resistance was also varied. A total of 864 islanding and 510 non-islanding cases were simulated and the three-phase energy values of terminal voltage and current were recorded for each simulation.

To make the data collection consistent, a 'trigger' signal was defined. It is a transient detector, which is based on the three phase energy value of the voltage waveform output through the band-pass filter in the range $312.5-650 \mathrm{~Hz}$ (defined as EV4). The selection of EV4 as the trigger was straightforward due to its quality of showing a comparatively large variation in the event of a transient. The trigger threshold was set as 0.4 by studying the dataset. Thus, the data was collected after a delay of $0.01 \mathrm{~s}$, if the value of EV4 exceeds 0.4 . The delay of $0.01 \mathrm{~s}$ allows the required energy extraction process to be completed.

\section{Classifier modelling}

The decision tree classifier was modelled based on the theory explained earlier, using the CART $®$ Pro V6 commercial software (Steinberg \& Colla, 1995). From the total dataset, which included a total of 864 islanding and 510 non-islanding cases, $67 \%$ was used as the learning data, while the remaining $33 \%$ was used as the testing data. The testing data were extracted randomly from each category of events described above to ensure testing against all types of transient events.

Table 3: Variable importance for initial DT with 8 variables

\begin{tabular}{cc}
\hline Variable & Importance $(\%)$ \\
\hline EV1 & 100.00 \\
EI4 & 20.60 \\
EV4 & 7.80 \\
EV3 & 5.88 \\
\hline
\end{tabular}

\begin{tabular}{cc}
\hline Variable & Importance (\%) \\
\hline EV2 & 1.26 \\
EI1 & 0 \\
EI2 & 0 \\
EI3 & 0 \\
\hline
\end{tabular}

Initially, a DT was built using eight variables (EV1, EV2, EV3, EV4, EI1, EI2, EI3 and EI4) corresponding to voltage (V) and current (I) waveforms through band-pass $(B P)$ filters in frequency ranges: i. $2500-5000 \mathrm{~Hz}(B P 1)$, ii. $1250-2500 \mathrm{~Hz}(B P 2)$, iii. $650-1250 \mathrm{~Hz}(B P 3)$ and iv. $312.5-650 \mathrm{~Hz}(B P 4)$. These variables were created by extracting the energies of the filtered waveforms as explained earlier. As stated in a previous section, in building a DT it is possible to obtain the usefulness of each variable in the classification process as a percentage of the 'best performing variable' which is shown in Table 3. According to Table 3, relevancy of the variables EI1, EI2, EI3 and EV2 are very low, and they were omitted in 


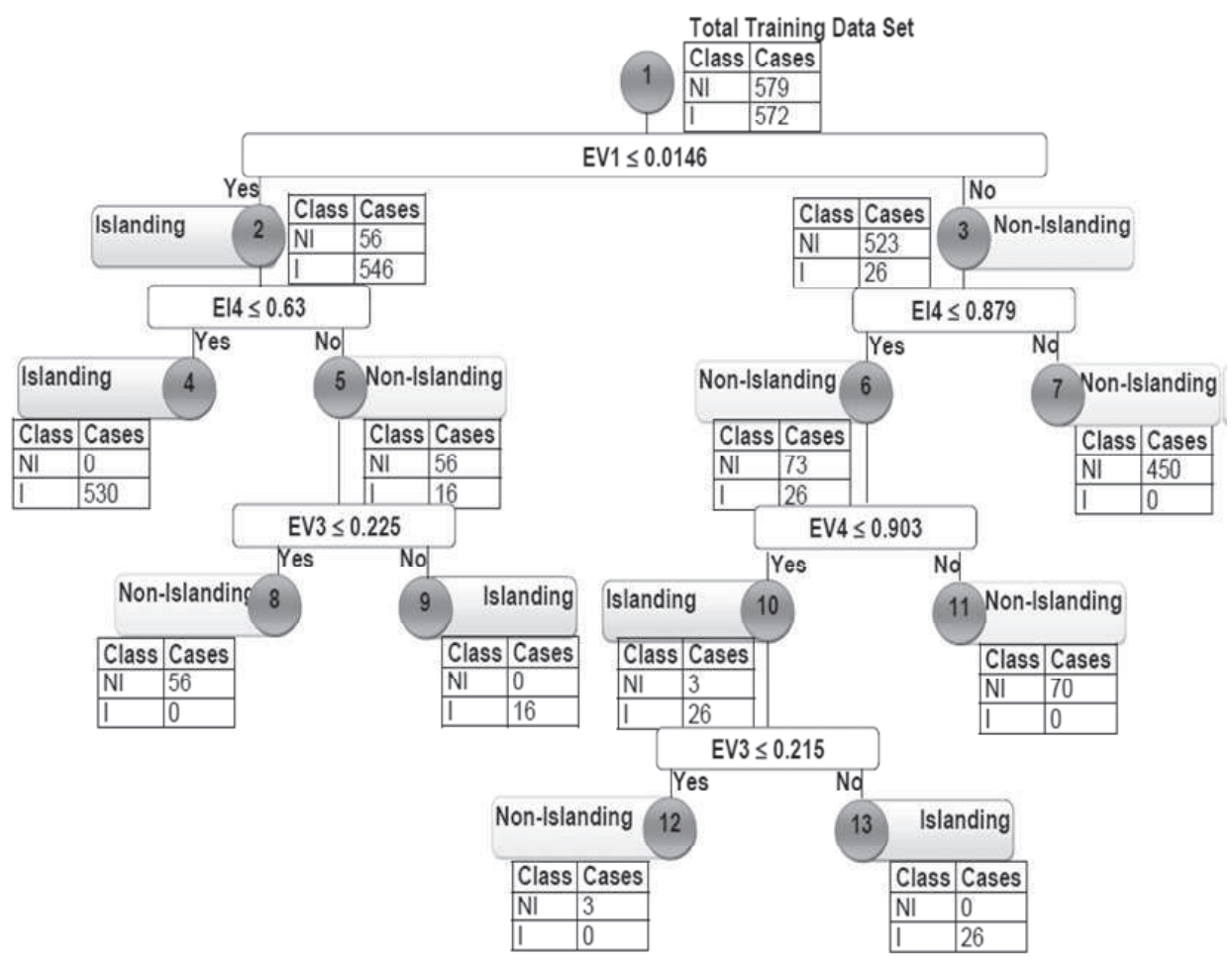

Figure 8: The decision tree classifier trained for detecting power islands

building the DT. This optimization reduced the number of variables from eight to four, which in turn significantly reduced the implementation cost and complexity with the reduction of the required number of filters.

Table 4: DT classification accuracies through CART software

\begin{tabular}{lcccc}
\hline Class & \multicolumn{2}{c}{ Training } & \multicolumn{2}{c}{ Testing } \\
& $\begin{array}{l}\text { Total } \\
\text { cases }\end{array}$ & $\begin{array}{l}\text { Correctly } \\
\text { classified }\end{array}$ & $\begin{array}{l}\text { Total } \\
\text { cases }\end{array}$ & $\begin{array}{l}\text { Correctly } \\
\text { classified }\end{array}$ \\
\hline $\begin{array}{l}\text { Islanding } \\
\text { Non- }\end{array}$ & 579 & $579(100 \%)$ & 292 & $292(100 \%)$ \\
$\begin{array}{l}\text { Islanding } \\
\text { Total }\end{array}$ & 572 & $572(100 \%)$ & 289 & $288(100 \%)$ \\
& 1151 & $1151(100 \%)$ & 581 & $580(100 \%)$
\end{tabular}

The optimized DT shown in Figure 8 returned the same classification accuracy as the initial DT, and the classification accuracies are given in Table 4. The overall accuracy (correct classification of both islanding and nonislanding events as a percentage of total events tested) of $100 \%$ was achieved in training the classifier. In testing, the overall accuracy achieved was $99.83 \%$, while giving $100 \%$ accuracy in classifying islanding events.

Figure 9 presents the simulation results observed at each point of signal processing from the voltage and current outputs (PT and CT outputs) measured at the DG terminal up to the classifier for a non-islanding (temporary three-phase fault with $1 \Omega$ fault impedance at the bus-2 in Figure 7) and an islanding (opening of breaker 'Tripgrid' in Figure 7) event. Figures 9(c) - (g) are intended to illustrate the signal processing in each step under feature extraction for a non-islanding (fault) and an islanding event.

Feature extraction involves band-pass filtering, rectifying, three phase adding and low pass filtering. Only the band-pass filter outputs of phase-a are shown for the two cases (non-islanding to the left and islanding to the right) in Figures 9(c) and (d). Similarly, the rectifier output is shown only for the frequency range $312.5<$ $\mathrm{f}<650 \mathrm{~Hz}$ (BP4) of phase-a voltage in Figure 9(e). The adder output is shown only for the BP4 frequency range in Figure 9(f). This comparison in Figures 9(c) to (g) depicts how the signal processing supports creating distinguishable differences (specifically in magnitude) in the features (EV1, EV3, EV4 and EI4) finally shown in 
Figure $9(\mathrm{~g})$ for the non-islanding and islanding events, which are the inputs to the DT classifier.

\section{Prototype implementation}

A prototype of the proposed islanding detection methodology was implemented in the laboratory level to investigate its real time performance. The main advantage in this proposed method compared to Lidula
\& Rajapakse (2010) arises in the implementation. Higher computational cost was avoided with the elimination of the DWT from feature extraction. In this proposing methodology, the feature extraction part was separately implemented using the actual filters. The output signals of the filters were used as inputs to the DT classifier, which was implemented on a microprocessor. The details of implementation of the feature extraction process and DT classifier are described in the following sub-sections. (a)

\section{3 phase fault with1@ fault}

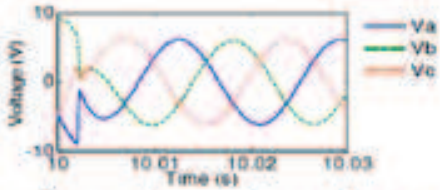

(b)

(c)

(d)

(e)
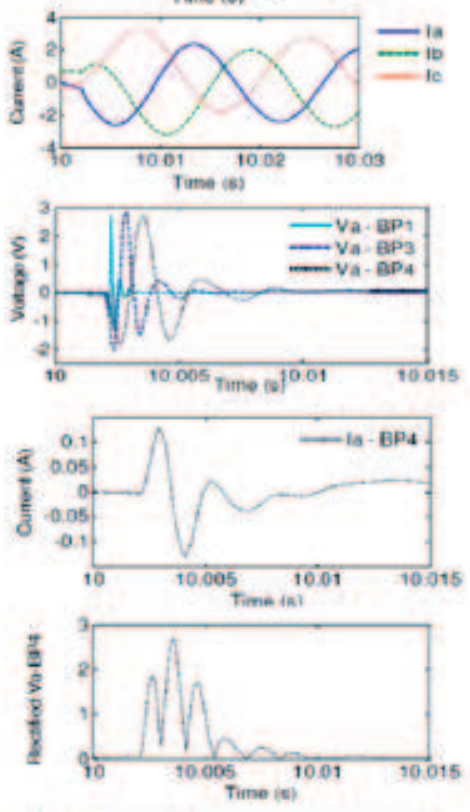

(f)

(g)
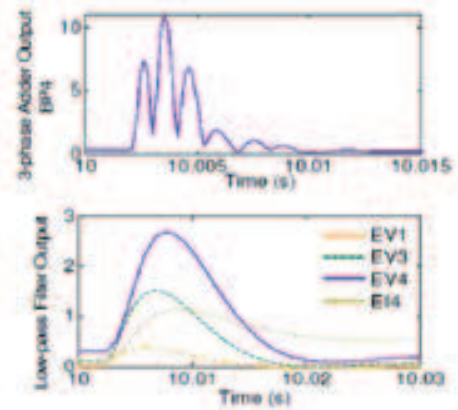

Non-Islanding
Opening breaker "TripGrid"
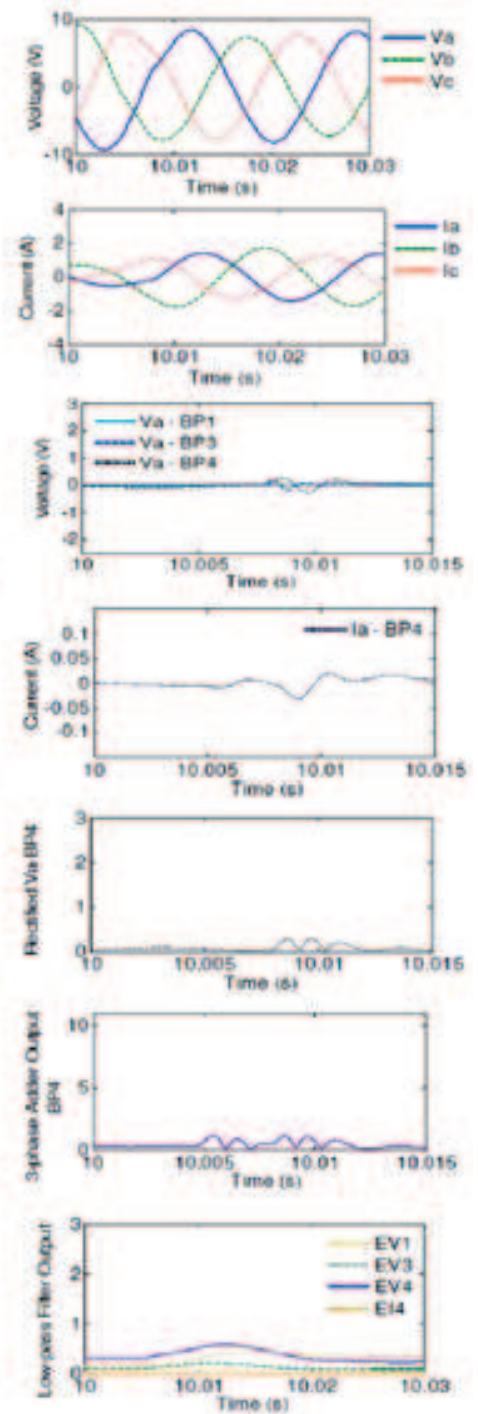

Islanding

Figure 9: The signal processing process in the proposed relay for a non-islanding and an islanding event: (a) terminal voltage, (b) terminal current, (c) band-pass filters' outputs of phase-a voltages, (d) 312.5 - $650 \mathrm{~Hz}$ band-pass filter (BP4) output of phase-a currents, (e) rectifier outputs of phase-a voltage in the range $312.5-650 \mathrm{~Hz}$ (BP4) (f) 3-phase adder output of voltages in the range $312.5-650 \mathrm{~Hz}$ band (BP4) and (g) low-pass filter outputs 
Table 5: Standard resistor values for the band-pass filters

\begin{tabular}{lcccccc}
\hline Frequency band & $\begin{array}{c}\mathrm{R}_{21} \\
(\mathrm{k} \Omega)\end{array}$ & $\begin{array}{c}\mathrm{R}_{11} \\
(\mathrm{k} \Omega)\end{array}$ & $\begin{array}{c}\mathrm{R}_{31} \\
(\mathrm{k} \Omega)\end{array}$ & $\begin{array}{c}\mathrm{R}_{22} \\
(\mathrm{k} \Omega)\end{array}$ & $\begin{array}{c}\mathrm{R}_{12} \\
(\mathrm{k} \Omega)\end{array}$ & $\begin{array}{c}\mathrm{R}_{32} \\
(\mathrm{k} \Omega)\end{array}$ \\
\hline $2500-5000 \mathrm{~Hz}(\mathrm{BP} 1)$ & 23.5 & 8.2 & 1.6 & 14.6 & 5.0 & 1.0 \\
$650-1250 \mathrm{~Hz}(\mathrm{BP} 3)$ & 97.6 & 33.2 & 6.04 & 61.9 & 21.0 & 3.83 \\
$312.5-650 \mathrm{~Hz}(\mathrm{BP} 4)$ & 174.0 & 59.0 & 12.4 & 110.0 & 37.4 & 8.06 \\
\hline
\end{tabular}

\section{Feature extraction}

As shown in Figure 1 and as discussed earlier, the feature extraction process begins with the band-pass filtering of the three phase voltages and currents. The estimated values for all the components of the fourthorder Butterworth band-pass filters (Figure 2) are given in Table 5. A total of 12 fourth-order band-pass filters (3 filters for each phase of voltage, and 1 filter for each phase of current) were implemented.

The next stage of feature extraction is the rectifier circuits. The brief discussion on rectifiers in a previous section shows that each rectifier circuit consists of two operational amplifiers, five resistors and two diodes. The selected values for the resistances are shown on Figure 3 (Gerstenhaber et al., 2010).

The third stage of the process is the adder circuit. The resistor values of the adder circuits $\left(R_{f}\right.$ and $\left.R\right)$ were selected to obtain the required amplification gains for voltage and current signals (4 and 30, respectively).

The final stage of the feature extraction is low-pass filtering. As discussed earlier second-order Butterworth filters were used considering their capability in giving smooth output with minimal distortions. The component values used for the low pass filters corresponding to Figure 6 were determined as shown in Table 6. The output from the four low-pass filters represents the four energy signals that act as predictors to the classifier.

Table 6: Standard capacitor and resistor values for the low-pass filters

\begin{tabular}{cccccc}
\hline $\begin{array}{c}\text { Cut-off } \\
\text { frequency }\end{array}$ & $\begin{array}{c}\mathrm{C} 1 \\
(\mathrm{nF})\end{array}$ & $\begin{array}{c}\mathrm{C} 2 \\
(\mathrm{nF})\end{array}$ & $\begin{array}{c}\mathrm{R}_{1} \\
(\mathrm{k} \Omega)\end{array}$ & $\begin{array}{c}\mathrm{R}_{2} \\
(\mathrm{k} \Omega)\end{array}$ & $\begin{array}{c}\mathrm{R}_{3} \\
(\mathrm{k} \Omega)\end{array}$ \\
\hline $50 \mathrm{~Hz}$ & 10 & 47 & 138.2 & 138.2 & 155.6 \\
\hline
\end{tabular}

\section{Classifier}

The classification algorithm was implemented using the Microchip PIC18F46K22 microcontroller. The key features of this microcontroller are given below.
- $64 \mathrm{~KB}$ flash programme memory

- 16 MIPS CPU speed

- 3,896 bytes RAM

- 1024 byte data EEPROM

- 2-A/E/USART,2-MSSP(SPI/I2C) digital communication peripherals

- $3 \times 8$-bit, $4 \times 16$-bit timers

- 28 ch, 10-bit ADC

The microcontroller was incorporated with a MPLAB ICD2 connector, resistors, a seven-segment display for testing and light emitting diodes for logic flags. The developed classification unit is shown in Figure 10. The required software modules for converting analog signals into digital values and displaying characters on a 4 digit seven segment display were developed. Furthermore, the subroutine for the DT classifier was directly imported from the CART software to programme the microchip.

The instantaneous energy values EV1, EV3, EV4 and EI4 resulting from the low-pass-filter outputs were fed to the microchip through the MPLAB ICD2 connector. The system first reads EV4 and compares the value to the trigger level (0.4). Once EV4 surpasses this value, a delay of $0.01 \mathrm{~s}$ is provided before collecting EV1, EV3, EV4 and EI4. These values are used as the input parameters for the DT classifier subroutine, which returns an integer representing the result of the classifier. Upon detection of an islanding event, an islanding flag is set to high, and the programme ends. If a non-islanding event occurs, the system waits for EV4 to decrease to a value below the trigger, and the process repeats until an island is detected.

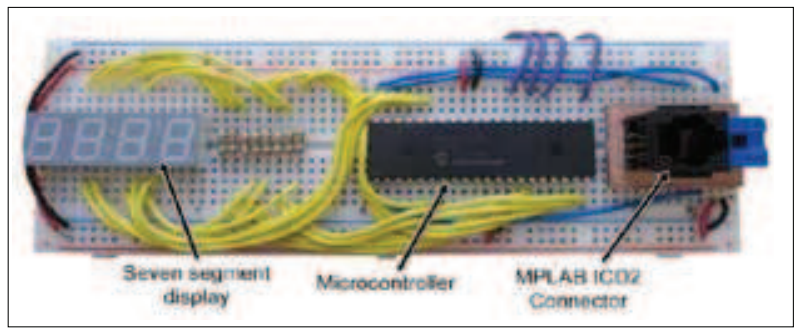

Figure 10: The classifier unit 


\section{Experimental setup}

The waveform generator, real-time playback (RTP) was used to generate the necessary three phase voltage and current signals to test the classification system. The recorded waveforms obtained from PSCAD/EMTDC simulation were played back via the RTP. A photograph of the experimental setup used for prototype testing is shown in Figure 11.

\section{RESULTS}

The implemented prototype transient based islanding detection relay was tested using 26 islanding and 26 nonislanding events.

\section{Validation of the experimental setup operation}

In order to ensure correct operation of the classifier, the analog waveforms generated from the simulations were compared against the actual waveforms observed in the implemented islanding detection relay. Figure 12 shows a comparison of the simulated and measured signals observed during an islanding transient. Figure 12(c) shows that the energy values obtained from the implemented actual filters are in total agreement with the simulated values.

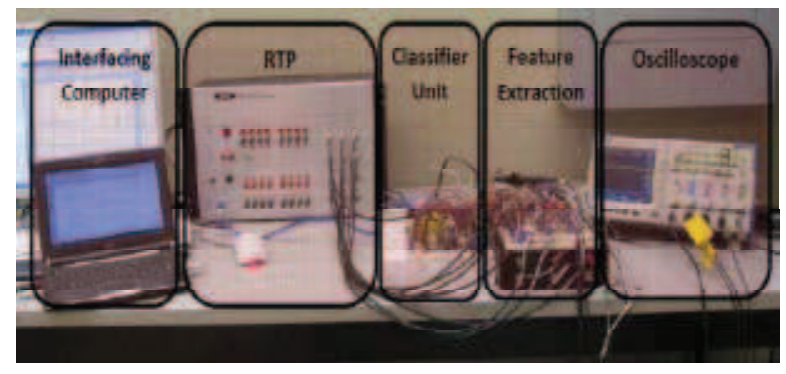

Figure 11: Experimental setup

\section{Classification results}

The operation of the implemented classifier was tested for 52 cases, which involved 26 islanding events and 26 non-islanding events. Figure 13 shows the performance

\section{Simulated}

(a)

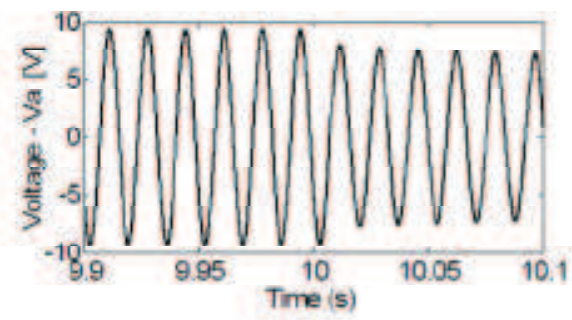

(b)

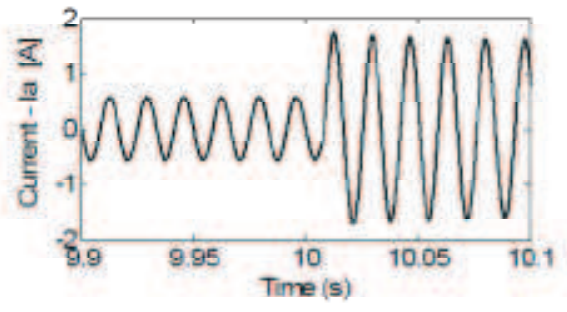

(c)

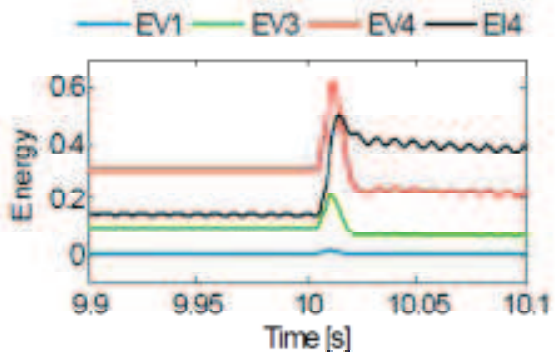

Measured
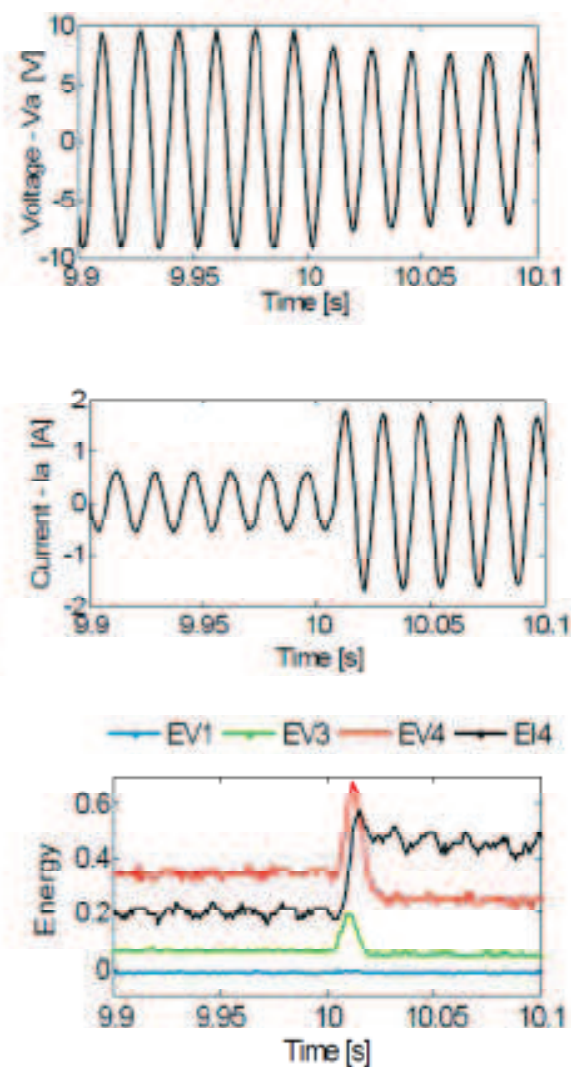

Figure 12: Comparison of simulated and measured signals (a) phase a-voltage, (b) phase-a current and (c) energy waveforms 


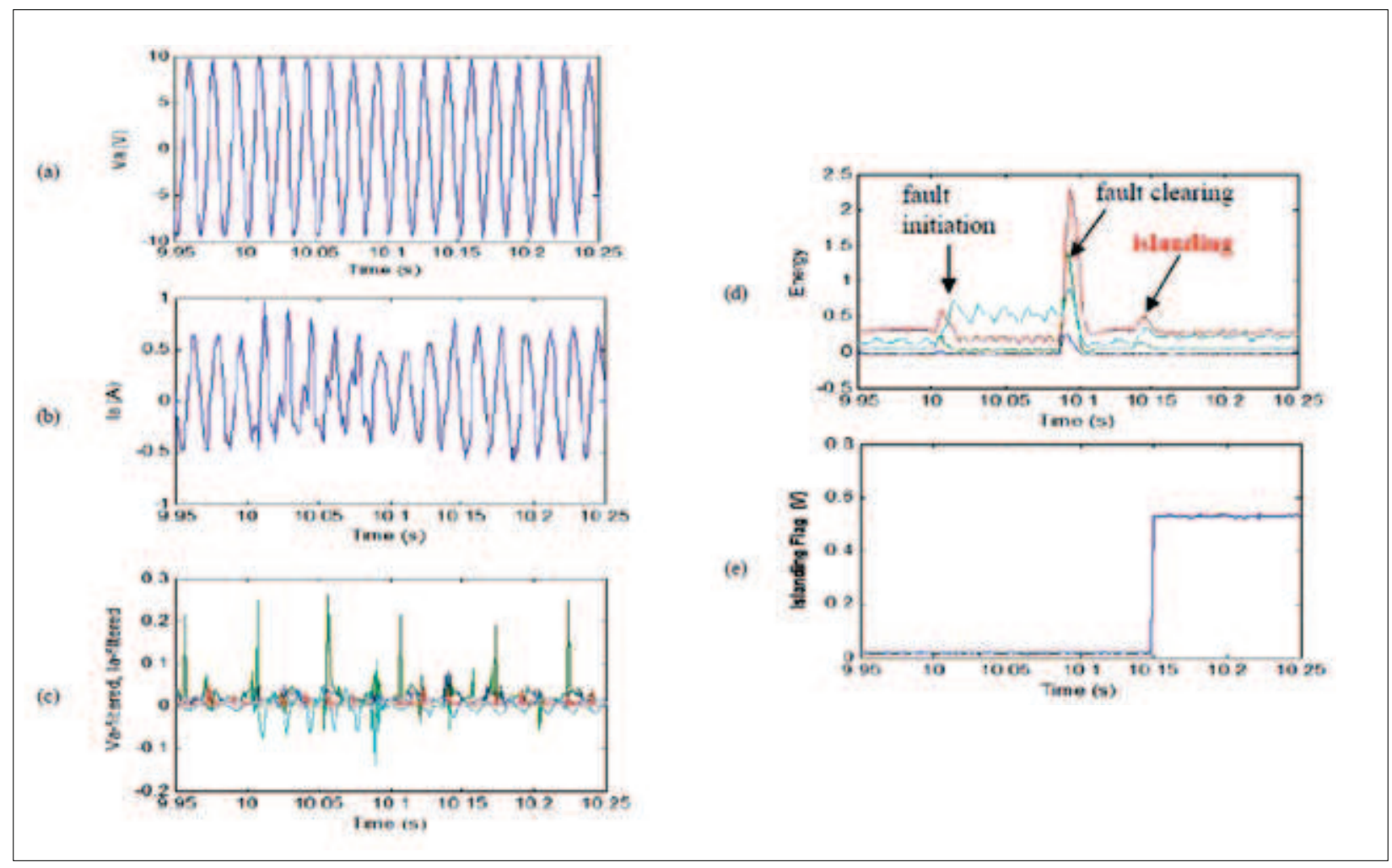

Figure 13: Observations at SPU (a) phase-a voltage, (b) phase-a current, (c) filtered voltage and current, (d) energy waveforms and (e) islanding flag

of the classifier during one of the islanding events, which happens after a temporary fault (two non-islanding events: fault initiation and fault clearing) when the total system load was $8 \mathrm{MW}$ at a 0.95 power factor. Figure 13(a) and (b) presents the measured phase-a voltage and phase-a current at the DG terminal in the real-time playback (RTP). Figure 13(c) presents the band pass filters' outputs of measured voltage and current signals. The extracted features EV1, EV3, EV4 and EI4 are shown in Figure 13(d) and the islanding flag of the implemented classifier is shown in Figure 13(e). Although it is not distinguishable from the measured voltage and current signals shown in Figures 13(a) and (b), Figure 13(d) clearly shows a significant change in the energy contents at the transients generated at the initiation of fault at $10 \mathrm{~s}$, fault clearing at $10.08 \mathrm{~s}$ and islanding at $10.15 \mathrm{~s}$. Based on the energy values observed, the classifier was able to correctly recognize the islanding transient out of the other fault transients.

The results observed with the implemented islanding detection relay for the 52 cases tested are summarized in Table 7. It shows that 24 out of the 26 islanding cases tested were correctly classified as islanding, and 25 out of the 26 non- islanding cases were correctly classified as non-islanding. This gives an overall classification accuracy of $94 \%$. The islanding cases that were incorrectly classified operated due to the transients originating from fault reflections. In average, the islanding detection time of the implemented relay was estimated as $14 \mathrm{~ms}$. The maximum detection time observed is only $19 \mathrm{~ms}$, which is less than 2 cycles.

Table 7: Experimental accuracy results

\begin{tabular}{lccccc}
\hline Class & \multicolumn{2}{c}{ Experiment } & \multicolumn{3}{c}{ Detection time (ms) } \\
& $\begin{array}{c}\text { Total } \\
\text { cases }\end{array}$ & $\begin{array}{c}\text { Correctly } \\
\text { classified cases }\end{array}$ & Min & Max & Average \\
\hline Islanding & 26 & $24(92 \%)$ & & & \\
$\begin{array}{l}\text { Non- } \\
\text { islanding }\end{array}$ & 26 & $25(96 \%)$ & 12 & 19 & 14 \\
Total & 52 & $49(94 \%)$ & & & \\
\hline
\end{tabular}

\section{CONCLUSION}

Development of a prototype islanding detection relay transient signals was presented in this paper. The islanding detection relay uses a decision tree classifier with the 
features extracted through a set of tuned analog band pass filters. The proposed islanding detection technique was not only successful in giving comparatively accurate classification results, but also presented a simplified design with reduced computational and implementation cost.

In testing the implemented prototype relay, classification accuracies matched very well with the simulated results. With the implemented relay, an overall accuracy of $94 \%$ was achieved in classifying islanding and non-islanding events correctly. For the cases tested, the average islanding detections time was only $14 \mathrm{~ms}$, which is less than one cycle and the maximum detection time observed was only $19 \mathrm{~ms}$, which is less than two cycles.

With its fast response, higher accuracy level, and the potential for low cost implementation, this methodology can be accepted as a promising technology to apply in microgrids, which require fast and reliable islanding detection (Katiraei et al., 2005) and could be a challenging substitute for highly reliable but expensive and complex transfer trip schemes (Etxegarai et al., 2011).

\section{REFERENCES}

1. Breiman L., Friedman J., Olshen R. \& Stone C. (1984). Classification and Regression Trees. Pacific Grove: Wadsworth Books, Belmont, California, USA.

2. Chowdhury S., Chowdhury S.P. \& Crossley P. (2009). Microgrids and active distribution networks. IEE Power and Energy Series 6. The Institute of Electrical Engineers, London, UK. DOI: http://dx.doi.org/10.1049/PBRN006E

3. El-Arroudi K., Joós G., Kamwa I. \& McGillis D.T. (2007). Intelligent-based approach to islanding detection in distributed generation. IEEE Transactions on Power Delivery 22: 828 - 835 .

4. Etxegarai A., Eguía P. \& Zamora I. (2011). Analysis of remote islanding detection methods for distributed resources, International Conference on Renewable Energies and Power Quality, ICREPQ'11, April 2011, pp. 1 - 6.

5. Freitas W., Huang Z. \& Xu W. (2005 a). A practical method for assessing the effectiveness of vector surge relays for distributed generation applications. IEEE Transactions on Power Delivery 20(1): $57-63$.

DOI: http://dx.doi.org/10.1109/TPWRD.2004.838637

6. Freitas W., Xu W., Affonso C.M. \& Haung Z. (2005 b). Comparative analysis between ROCOF and vector surge relays for distributed generation applications. IEEE Transactions on Power Delivery 20(2): 1315 - 1324. DOI: http://dx.doi.org/10.1109/TPWRD.2004.834869

7. Funabashi T., Koyanagi K. \& Yokoyama R. (2003). A review of islanding detection methods for distributed resources. Proceedings of the Power Tech Conference, Bologna, Italy, volume 2, pp. 6 - 11 .

8. Gerstenhaber M. \& Malik R. (2010). More value from your absolute value circuit-difference amplifier enables lowpower, high-performance absolute value circuit. Analog Dialogue, volume 44. Available at www.analog.com/ library/analogdialogue/archives/44-4/absolute.pdf

9. Hung G., Chang C.C. \& Chen C.L. (2003). Automatic phase-shift method for islanding detection of gridconnected photovoltaic inverters. IEEE Transactions on Energy Conversion 18(1): 169 - 173.

DOI: http://dx.doi.org/10.1109/TEC.2002.808412

10. IEEE Standard 1547-2003 (2003). IEEE Standard for Interconnecting Distributed Resources with Electric Power Systems, pp. 1 - 16. IEEE Standards Association, New Jersy. USA.

11. Jenkins N., Allan R., Crossley P., Kirschen D. \& Strbac G. (2000). Embedded Generation, IEE Power and Energy Series 31. The Institute of Electrical Engineers, London, UK.

12. Katiraei F., Iravani M.R. \& Lehn P.W. (2005). Micro-grid autonomous operation during and subsequent to islanding process. IEEE Transactions on Power Delivery 20 (1): $248-257$.

DOI: http://dx.doi.org/10.1109/TPWRD.2004.835051

13. Kim J.E. \& Hwang J.S. (2000). Islanding detection method of distributed generation units connected to power distribution system, Proceedings of the International Conference on Power System. PowerCon 2000, Perth, Western Australia, pp. 643 - 647.

14. Kugelstadt T. (2002). Active filter design techniques. $O p$ Amps for Everyone (ed R. Mancini). Available at http:// www.focus.ti.com/lit/an/slod006b/slod006b.pdf

15. Lidula N.W.A. \& Rajapakse A.D. (2010). A pattern recognition approach for detecting power islands using transient signals-part I: design and implement. IEEE Transactions on Power Delivery 25(4): 3070 - 3077. DOI: http://dx.doi.org/10.1109/TPWRD.2010.2053724

16. Lidula N.W.A. \& Rajapakse A.D. (2011). Microgrids research: a review of experimental microgrids and test systems. Renewable and Sustainable Energy Reviews Journal 15: 186 - 202.

DOI: http://dx.doi.org/10.1016/j.rser.2010.09.041

17. Mahat P., Chen Z. \& Bak-Jensen B. (2008). Review of islanding detection methods for distributed generation, Proceedings of the $3^{\text {rd }}$ International Conference on Electric Utility Deregulation and Restructuring and Power Technology (DRPT), pp. $2743-2748$.

18. Mahat P., Chen Z. \& Bak-Jensen B. (2009). A hybrid islanding detection technique using average rate of voltage change and real power shift. IEEE Transactions on Power Delivery 24(2): 764 - 771. DOI: http://dx.doi.org/10.1109/TPWRD.2009.2013376

19. O'Kane P. \& Fox B. (1997). Loss of mains detection for embedded generation by system impedance monitoring. Proeedings of the $6^{\text {th }}$ International Conference on 
Developments in Power Systems Protection, pp. 95 - 98. DOI: http://dx.doi.org/10.1049/cp:19970037

20. Pham J.P., Denboer N., Lidula N.W.A., Perera N. \& Rajapakse A.D. (2011). Hardware implementation of an islanding detection approach based on current and voltage transients, Proeedings of the IEEE Electrical Power and Energy Conference (IEEE EPEC), 4 October.

21. Redfern M.A., Usta O. \& Fielding G. (1993). Protection against loss of utility grid supply for a dispersed storage and generation unit. IEEE Transactions on Power Delivery 8(3): 948 - 954.

DOI: http://dx.doi.org/10.1109/61.252622

22. Ropp M.E., Begovic M. \& Rohatgi A. (1999). Analysis and performance assessment of the active frequency drift method of islanding prevention. IEEE Transactions on Energy Conversion 14(3): 810 - 816.

DOI: http://dx.doi.org/10.1109/60.790956
23. Salman S.K. \& King D.J. (2006). Comparison between the performance of ANN-based loss of mains relays using multilayer and non-layered perceptron approaches. International Journal of Innovations in Energy Systems and Power 1(1): $24-28$.

24. Steinberg D. \& Colla P. (1995). CART: Tree-Structured Non-Parametric Data Analysis. Salford Systems, San Diego, USA.

25. Walling R.A. \& Miller N.W. (2002). Distributed generation islanding implications on power system dynamic performance, Proceedings of the Power Engineers Society Summer Meeting, IEEE, volume 1, Chicago, USA, 25 July, pp. $92-96$.

26. Xu W., Mauch K. \& Martel S. (2004). An Assessment of DG Islanding Detection Methods and Issues for Canada. CANMET Energy Technology Centre, Natural Resources Canada. 